\title{
A new approach for inversion of receiver function for crustal structure in the depth domain
}

\author{
TianYu Zheng ${ }^{1}$, YuMei He ${ }^{1 *}$, and Yue Zhu ${ }^{1,2}$ \\ ${ }^{1}$ Key Laboratory of Earth and Planetary Physics, Institute of Geology and Geophysics, Chinese Academy of Sciences (CAS), Beijing 100029, China; \\ 2University of Chinese Academy of Sciences, Beijing 100049, China
}

\section{Key Points:}

- A new approach for imaging crustal structure from inversion of stacking receiver function amplitude in the depth domain is developed.

- Numerical tests verify the accuracy and effectiveness of the new inversion method.

- Compared with the waveform inversion of the receiver function, the new method weakens the tendency to run into the local extreme.

Citation: Zheng, T. Y., He, Y. M., and Zhu, Y. (2022). A new approach for inversion of receiver function for crustal structure in the depth domain. Earth Planet. Phys., 6(1), 83-95. http://doi.org/10.26464/epp2022008

\begin{abstract}
A method for reconstructing crustal velocity structure using the optimization of stacking receiver function amplitude in the depth domain, named common conversion amplitude (CCA) inversion, is presented. The conversion amplitude in the depth domain, which represents the impedance change in the medium, is obtained by assigning the receiver function amplitude to the corresponding conversion position where the P-to-S conversion occurred. Utilizing the conversion amplitude variation with depth as an optimization objective, imposing reliable prior constraints on the structural model frame and velocity range, and adopting a stepwise search inversion technique, this method efficiently weakens the tendency of easily falling into the local extremum in conventional receiver function inversion. Synthetic tests show that the CCA inversion can reconstruct complex crustal velocity structures well and is especially suitable for revealing crustal evolution by estimating diverse velocity distributions. Its performance in reconstructing crustal structure is superior to that of the conventional receiver function imaging method.
\end{abstract}

Keywords: crustal imaging; receiver function; depth domain; inversion

\section{Introduction}

Since Earth's formation, its crustal structure has been repeatedly modified by structural, magmatic, and metamorphic processes. This long-term evolution has complicated the structure of the crust, but it has also left traces of the evolutionary process. Studying continental evolution by using imprints remaining in the crust is of great importance. In the past several decades, many efforts have been made to obtain detailed crustal structures from nearvertical and wide-angle seismic reflection surveys. With the development of seismic observation technology, a large number of high-fidelity seismic recordings have been collected at densely distributed stations with large-scale coverage and high degrees of spatial resolution. Using earthquake propagation information, we can image large areas of the Earth's interior structure. Receiver function imaging technology, which considers teleseismic earthquakes as natural energy sources, has been widely used to determine the crustal structure beneath seismic stations.

As teleseismic P-waves travel through the Earth's interior, part of

Correspondence to: Y. M. He, ymhe@mail.igcas.ac.cn

Received 11 AUG 2021; Accepted 04 OCT 2021.

Accepted article online 23 NOV 2021.

C 2022 by Earth and Planetary Physics. the P-wave energy is converted into S-wave energy at various velocity discontinuities. To deconvolve the vertical component from the horizontal components of the teleseismic waveform, in order to remove source effects, a time series named "the receiver function" is produced (Langston, 1979). The receiver function isolates the P-to-S (Ps) converted phases at the interfaces within the crust and upper mantle and allows the travel times from the interfaces to the surface to be determined. These Ps-converted phases strongly constrain the depth and velocity contrasts of discontinuities.

As a conventional waveform analysis technique, the inversion of the receiver function applies stacked waveforms as an optimization objective to estimate a crustal velocity model beneath the station. However, receiver function waveform inversion involves an inherent nonuniqueness known as the velocity-depth ambiguity due to significant tradeoffs between the absolute shear wave velocity and the corresponding depth under the same travel time difference (Ammon et al., 1990). The introduction of a priori information is necessary to suppress this problem. The joint inversion method of receiver function and surface wave dispersion, in which the surface wave dispersion measurements constrain the regional average velocity, is developed to reduce the nonuniqueness of the problem (Julià et al., 2000; Chang et al., 2004). 
However, the weakness of easily falling into the local extremum trap still exists in joint inversion. Meanwhile, the resolution of surface wave dispersion is relatively low, which hinders the joint method from revealing complex intracrustal structures.

Referring to the data processing techniques used in reflection seismology, researchers have developed time-depth conversion and stacking methods in receiver function imaging (e.g., Dueker and Sheehan, 1997; Zhu LP, 2000; Sheehan et al., 2000; Poppeliers and Pavlis, 2003; Chen $L$ et al., 2005). The common conversion point (CCP) stacking technique is representative of such techniques (Dueker and Sheehan, 1997). By assuming a velocity model and assigning the amplitude of the Ps phase to the corresponding conversion position, and stacking multiple events with the conversion within a bin (Figure 1), the topography of the velocity discontinuity along a section is illuminated directly. Using this relatively intuitive approach, the major velocity discontinuities in the crust and upper mantle, including the Moho, lithosphereasthenosphere boundary, 410-km interface, and 660-km interface, can be visually identified (e.g., Kind et al., 2002; Gilbert et al., 2003; Chen L et al., 2006; Lawrence and Shearer, 2006; Zheng TY et al., 2009, 2020; Ford et al., 2010; Tao K et al., 2014). However, inaccurate a priori velocity models, especially unreal sedimentary structures, would lead to considerably inaccurate migration solutions. Moreover, multiple reflections from the surface would produce apparent discontinuities and blur real interfaces. It has thus been difficult to distinguish complex intracrustal structures.

To build a reliable model space to avoid the trapping of local extrema in conventional receiver function waveform inversion, Zheng TY et al. (2015) and He YM et al. (2018) identified real intracrustal interfaces by stepwise forward modeling of CCP imaging from sedimentary cover to the lower crust. Dual constraints from the CCP stacking images and waveform characteristics enhance the reliability of receiver function imaging. However, in this way, only qualitative comparisons of observed and synthetic CCP are used as an a priori constraint on the main interface depth range, and detailed intracrustal structures are still missing.

Using the stacked receiver function amplitude at the common conversion position in the depth domain (hereafter referred to as

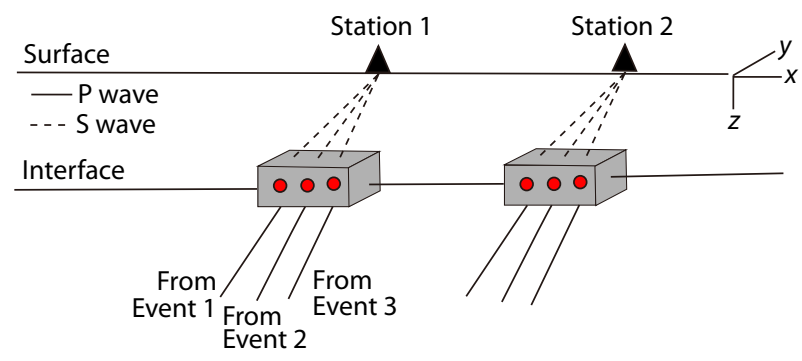

Figure 1. Schematic illustrations of receiver function stacking in the depth domain. The red circles mark the positions of conversion points. The amplitude of the receiver function converging in the gray bin is stacked and averaged to obtain the common conversion amplitude (CCA). The CCA represents the impedance contrast of the medium in the bin. The upper right corner shows the coordinate system of the structural section, where $x$ is along the profile and $z$ is vertically downward from the surface. the common conversion amplitude, CCA) as an inversion objective, we develop a new approach to image the detailed crustal structure. By quantitatively analyzing the conversion amplitude in the depth domain and constructing a considerably more accurate model space before each inversion, this approach efficiently suppresses the disadvantage of falling into the local extremum of waveform inversion in conventional receiver function imaging and has the potential to reveal complex intracrustal structures. We describe the principle and processing technology of the CCA inversion method in Section 2. Using numerical experiments, we estimate the accuracy and effectiveness of the CCA inversion method in Section 3 and discuss its resolution and limitations in Section 4. In Section 5, as an application example of the method, CCA inversion is implemented to reveal the detailed crustal structure along a portable seismic profile on the Liaodong Peninsula, Northeast China.

\section{CCA Inversion Method}

In the CCA inversion method, we directly use the stacked receiver function amplitude at the common conversion position in the depth domain as the inversion objective to estimate the detailed crustal structure beneath a dense seismic array. CCA, as a function of the variation in impedance with depth, allows one to obtain an intuitive estimation of an a priori structural model frame before inversion. The inversion is thus carried out step by step from the surface down to avoid the interference of multiple waves. The ability to build a reliable and considerably accurate model space before inversion helps to avoid the trapping of local minima. In addition, to overcome the low-frequency defect of the joint inversion of receiver function and surface wave data, we take the average velocity deduced from the surface-wave dispersion as a prior constraint of the velocity model frame.

\subsection{Construction of the Observation CCA}

Construction of the observation CCA is the first step in CCA inversion. We generate a receiver function using a time-domain maximum entropy deconvolution method (Wu QJ and Zeng RS, 1998). We then choose receiver functions within a given narrow range (in general less than $30^{\circ}$ ) of back azimuths to ensure that these receiver functions are sampling an identical receiver-side structure. By assuming a velocity model and using time-depth conversion, the amplitude of the Ps phase of the receiver function is assigned to the corresponding spatial location where the conversion occurred. Then, we divide the depth into bins and average the amplitude assigned in the same bin to calculate the CCA. The vertical dimension of the bin is determined by the sampling rate of the receiver function. The horizontal position of CCA sampling is determined by the ray path. This average amplitude of the receiver function represents the velocity change or, more precisely, the impedance change of the medium at the conversion position. CCA is thus a position function of the impedance characteristics of the medium.

To elucidate the inherent characteristics of CCA, we present CCA examples produced from synthetic receiver functions generated by simple or complicated crustal velocity models (Figure 2). As shown in Figure 2, a positive value of CCA indicates an upward decrease in velocity, and a negative value indicates an upward in- 

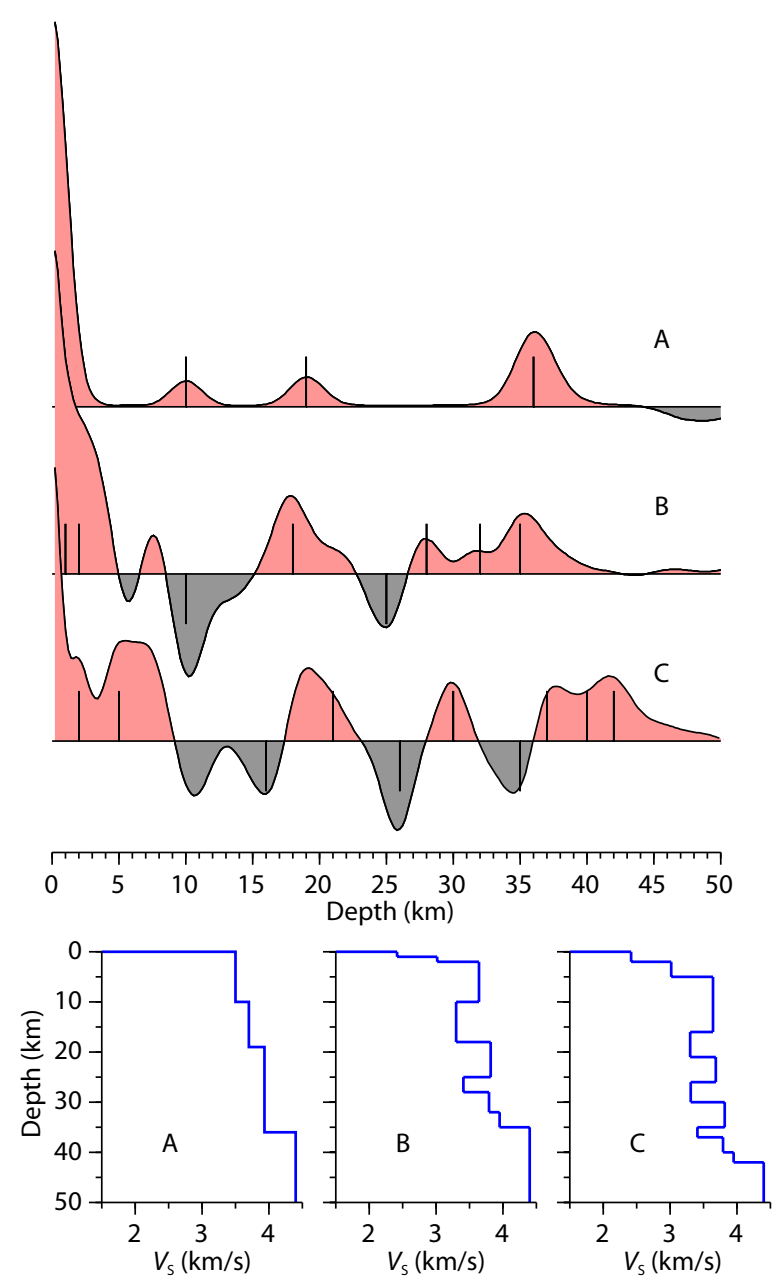

Figure 2. Top panel: CCA deduced from synthetic receiver functions generated by the velocity models (see bottom panel) and the epicentral distances and azimuth angles listed in Tables S1 (A) and S2 ( $B$ and $C$ ). Each depth of the velocity discontinuities of the velocity models is marked with a black line segment, where the upward velocity decrease/increase corresponds to the positive/negative amplitude of CCA. Bottom panel: the corresponding velocity models $A, B$ and $C$.

crease in velocity. The depth of discontinuity roughly corresponds to a positive/negative peak value of CCA, and the variation in velocity is related to the amplitude of CCA. The CCA function takes spatial position as an independent variable to express the change in spatial parameters directly. Therefore, the approximate depths and velocity change polarity of interfaces can be directly identified from CCA. This feature enables CCA to impose relatively accurate prior constraints on the structural model frame before each inversion. Conversely, in conventional receiver function waveform inversion, the objective function takes time as an independent variable, and the interface position cannot be directly estimated from the amplitude over time.

\subsection{Establishment of Objective Function and Nonlinear Inversion}

CCA inversion with prior constraints on the structural model frame and velocity range is a nonlinear and multiparameter op- timization problem. Taking CCA as the fitting target, we used an adapted hybrid global inversion method for each velocity structure inversion (Liu PC et al., 1995; Ai YS et al., 1998). In the CCA inversion, $O(z)$ is the observation value of $C C A, Y(z)$ is the theoretical value of CCA for each station, and $z$ refers to depth. $Y(z)$ is produced from synthetic receiver functions, which are generated from the estimated structural model and the ray parameters and azimuth angles of the same event-station pairs that generated $O(z)$. Synthetic seismograms are calculated by the reflection matrix method (Kennett, 1983). In the CCA inversion, the best fitting between $Y(z)$ and $O(z)$ of each station is searched by minimizing the objective function

$$
\text { OBJ }=1-\left[2 \sum_{i=1, n} O\left(z_{i}\right) \cdot Y\left(z_{i}\right)\right] /\left[\sum_{i=1, n} O^{2}\left(z_{i}\right)+\sum_{i=1, n} Y^{2}\left(z_{i}\right)\right] .
$$

This function measures the degree of coincidence between $O(z)$ and $Y(z)$ along the specific depth. Before starting a global searching process, a model framework - including the number of layers and the range of values dominating model parameters for each layer - needs to be specified. Due to the intuitiveness of the CCA, the model framework can be directly built from $O(z)$. For imaging crustal structure, $S$ velocity, layer thickness, and the $V_{p} / V_{s}$ ratio are selected as the dominant model parameters.

An a priori model is used as a migration model to produce the initial $O(z)$, and then a model framework is estimated. In the following processes, a series of acceptable models are generated to calculate synthetic receiver functions and are used as migration models for generating subsequent $O(z)$ and $Y(z)$. Finally, the resulting model is obtained by searching the minimum objective function.

\subsection{Key Techniques During CCA Inversion}

Based on the characteristics of converted wave propagation and receiving function waveforms, the following processing techniques should be considered. (1) Given that the multiple PpPs and PsPs + PpSs waves generated by shallow structures seriously interfere with the identification of real velocity discontinuities, it is important to use a stepwise inversion from the surface down to avoid the interference of multiple waves. (2) Due to the obvious differences in converted wave amplitudes between the Moho and other interfaces within the crust, we obtain a detailed estimate of the objective function by adding different weight values (of $0.1-1.0$ ) in different depth ranges. Generally, we set a weight of 1.0 for CCAs in the target depth range, a weight of 0.3 for CCAs above the target depth and 0.1 for those below the target depth. (3) Considering that the detailed changes in crustal material properties in a tectonic unit should be affected by the same geologic evolution, partitioned smooth velocity models are more suitable to yield reasonable results while avoiding individual abnormal distortions. An extra procedure is thus applied to the CCA inversion. First, station models with similar structural characteristics are combined to construct a regional smooth velocity model. Then, with fixed $V_{s}$ and $V_{p} / V_{s}$ for each layer from the average velocity model of a subregion, only the layer thicknesses are redetermined through the inversion for each station. We explain the process in detail through numerical testing in the next section. 


\section{Numerical Experiments on the Synthetic Imaging System}

The accuracy and effectiveness of the CCA inversion method are tested by numerical experiments. We first designed Model A (Figure 3a, Table 1), which is a simple 150-km-long multilayered crustal model and consists of 3 intracrustal layers with velocity increasing downward. The test system has 13 receivers (T01-T13) and 15 teleseismic events with epicentral distances in the range of $42^{\circ}-87^{\circ}$ and back azimuths within the range of $109^{\circ}-138^{\circ}$ (Table S1). The receivers are located in an N-S direction along $99.0^{\circ} \mathrm{E}$ and from $24.0^{\circ} \mathrm{N}$ to $25.2^{\circ} \mathrm{N}$ with station spacing of $0.1^{\circ}$. The epicenter locations are defined by reference to teleseismic events during 2011-2013. The earthquake catalog is listed in Table S1. According to the velocity models and the locations of sources and receivers, we calculate the receiver functions. The data were bandpass
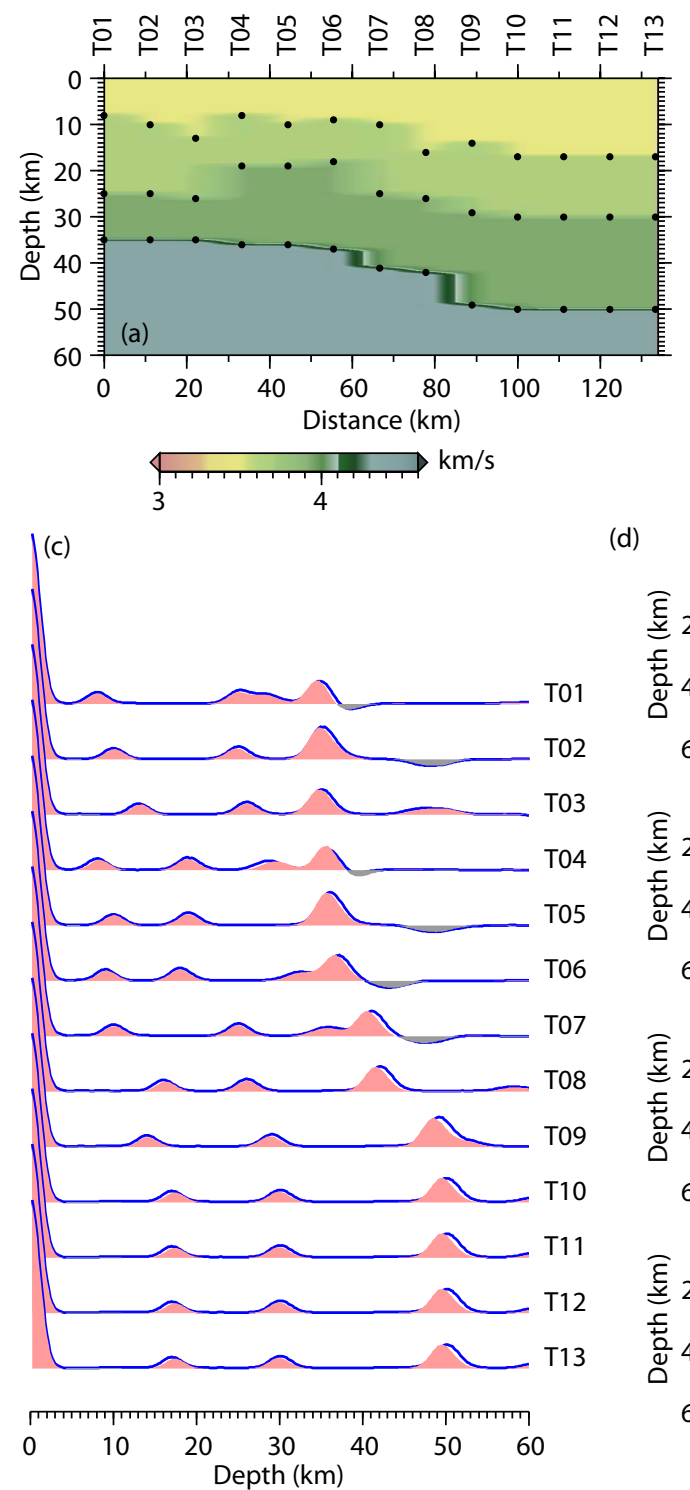

(d)

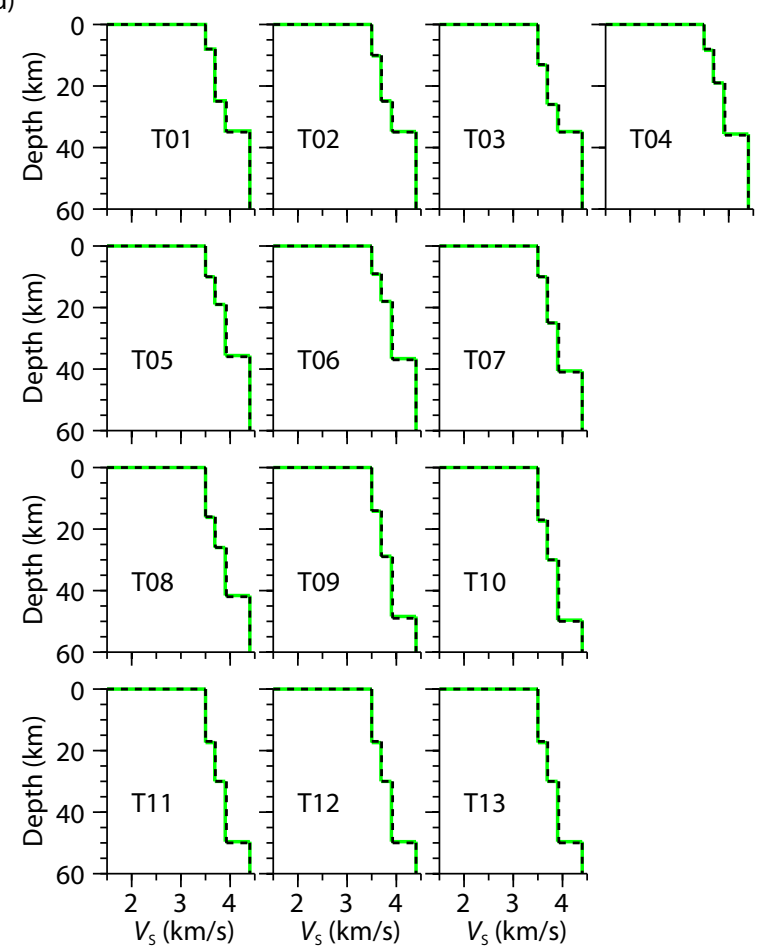

Figure 3. (a) S velocity structures of test Model A; interface depth of the test model is marked by black dots, and receiver names are labeled at the top of the plots. (b) CCP stacking images of the test Model A. (c) Comparison between the $O(z)$ (blue lines) obtained from the test system and $Y(z)$ (marked in pink and gray) resulting from the CCA inversion. Station names are labeled on the right. (d) Comparison between the $V_{s}$ velocity models of the test system (black dashed lines) and those resulting from the CCA inversion (green dashed lines); the station names are labeled in the graphs. 
Table 1. Comparison of velocity parameters between test Model A and inversion results.

\begin{tabular}{|c|c|c|c|c|c|c|c|c|c|}
\hline \multirow{2}{*}{ Layer } & \multicolumn{2}{|c|}{$V_{s}(\mathrm{~km} / \mathrm{s})$} & \multirow{2}{*}{$\begin{array}{l}\text { err } \\
\text { (\%) }\end{array}$} & \multicolumn{2}{|c|}{$V_{\mathrm{p}} / V_{\mathrm{s}}$} & \multirow{2}{*}{$\begin{array}{l}\text { err } \\
\%)\end{array}$} & \multicolumn{2}{|c|}{ Depth (km) } & \multirow{2}{*}{$\begin{array}{l}\text { err } \\
(\%)\end{array}$} \\
\hline & Mod & Syn & & Mod & Syn & & Mod & Syn & \\
\hline L1 & 3.50 & 3.50 & 0.0 & 1.74 & 1.74 & 0.2 & $8.0-17.0$ & $8.1-17.4$ & $0.9-4.1$ \\
\hline L2 & 3.70 & 3.69 & 0.3 & 1.73 & 1.76 & 1.5 & $18.0-30.0$ & $18.0-29.9$ & $0.0-0.8$ \\
\hline L3 & 3.93 & 3.90 & 0.9 & 1.73 & 1.75 & 1.2 & $35.0-50.0$ & $34.6-49.5$ & $0.7-1.2$ \\
\hline
\end{tabular}

Note: Mod - model parameters, Syn — resulting parameters from inversion, err — relative error.

models well (Figure 3d).

To fully test the ability to reconstruct complex crustal structures, we designed a more complex Model B with multiple low-velocity zones (LVZs) and various Moho depths (Figure 4a), referring to the most complex crustal structure in southeastern Tibet revealed
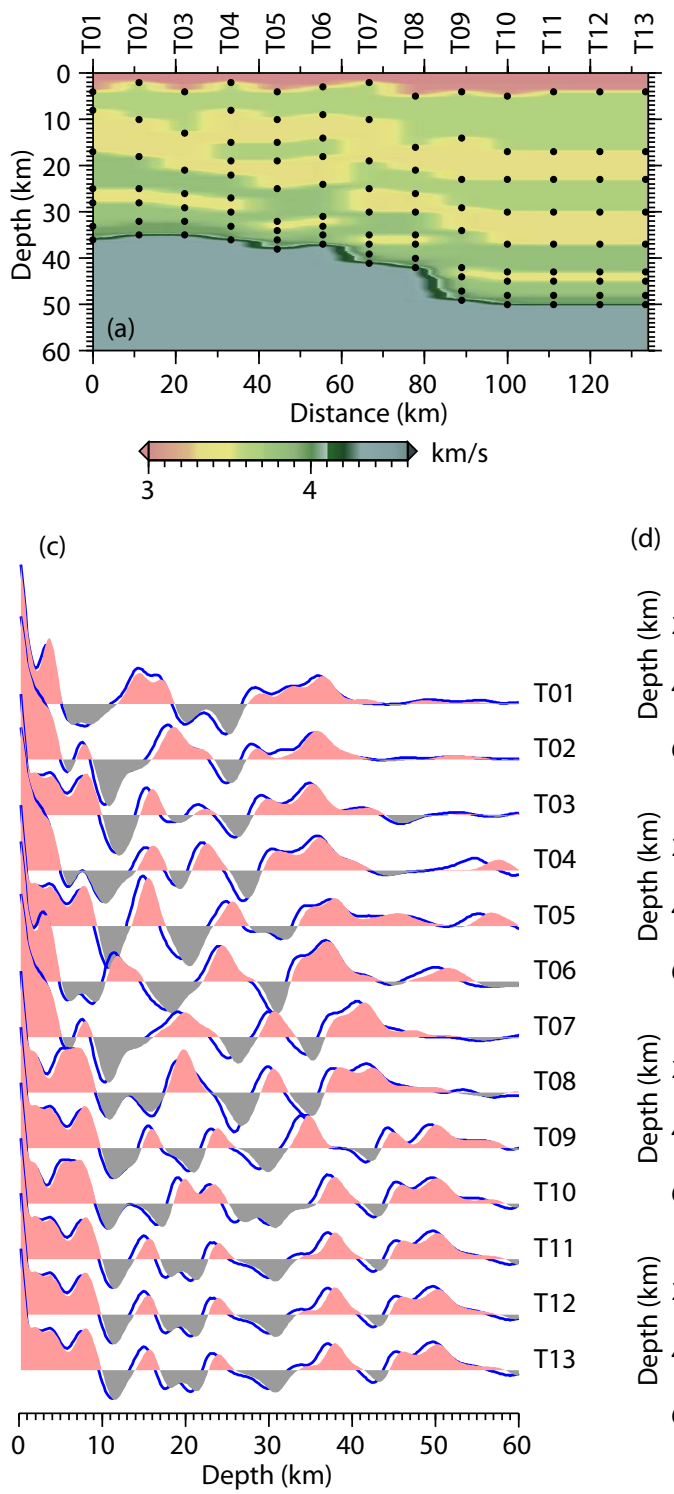

(d)
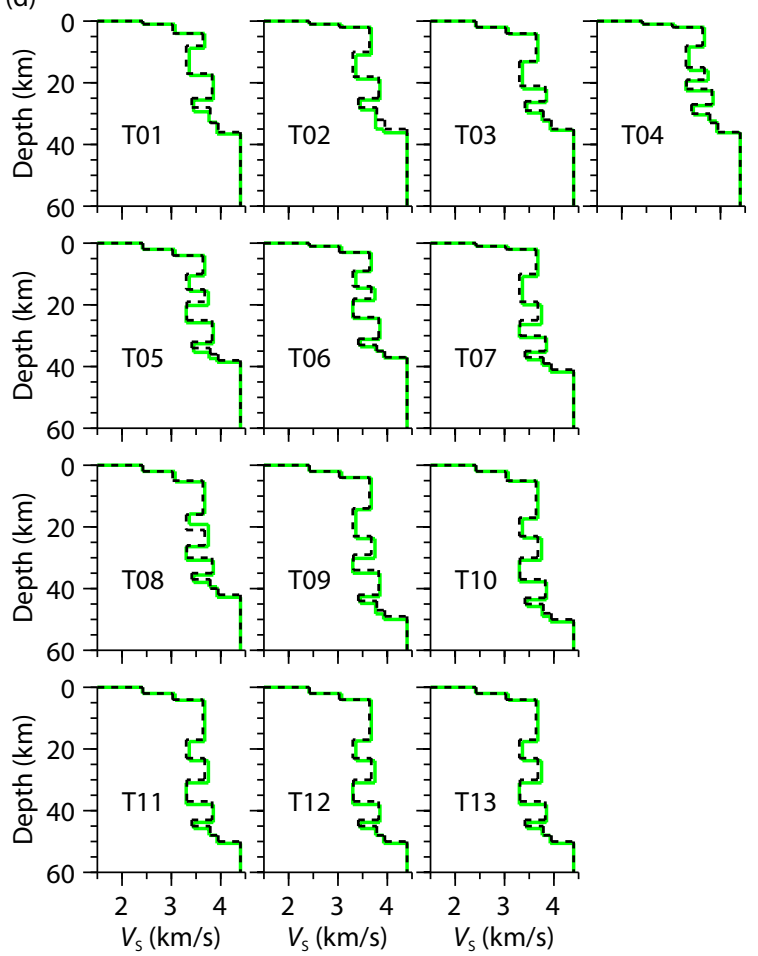

Figure 4. (a) S velocity structures of test Model B; interface depth of the test model is marked by black dots, and receiver names are labeled at the top of the plots. (b) CCP stacking images of the test Model B. (c) Comparison between the $O(z)$ (blue lines) obtained from the test system and $Y(z)$ (marked in pink and gray) resulting from the CCA inversion. Station names are labeled on the right. (d) Comparison between the $V_{s}$ velocity models of the test system (black dashed lines) and those resulting from the CCA inversion (green dashed lines); the station names are labeled in the graphs. 
Table 2. Comparison of velocity parameters between test Model $B$ and inversion results.

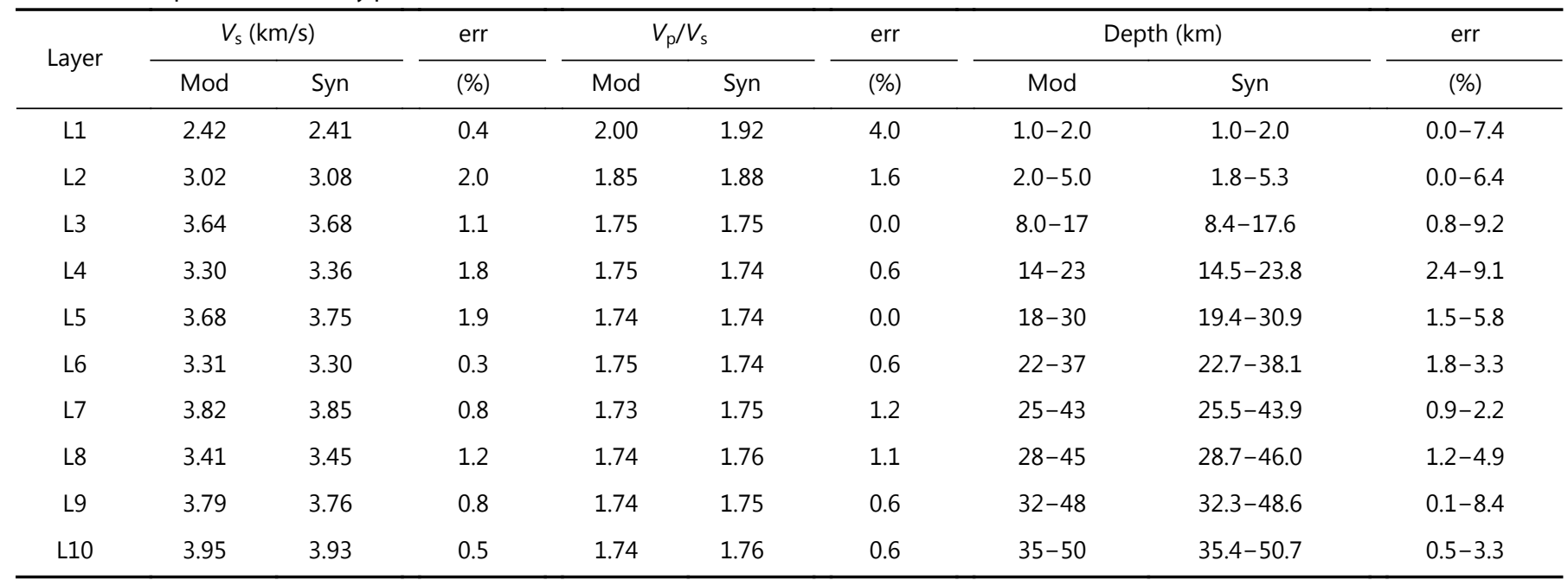

Note: Mod — model parameters, Syn — resulting parameters from inversion, err — relative error.

(a)

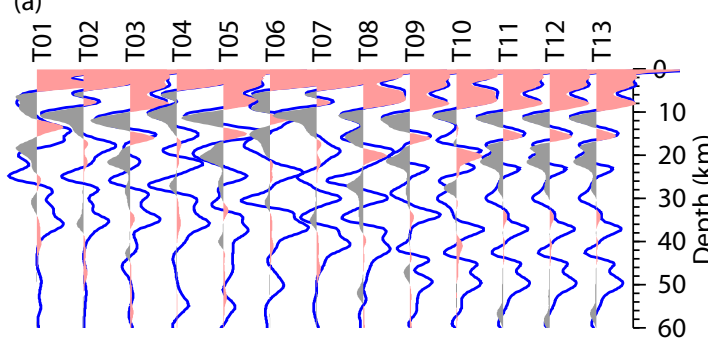

(b)

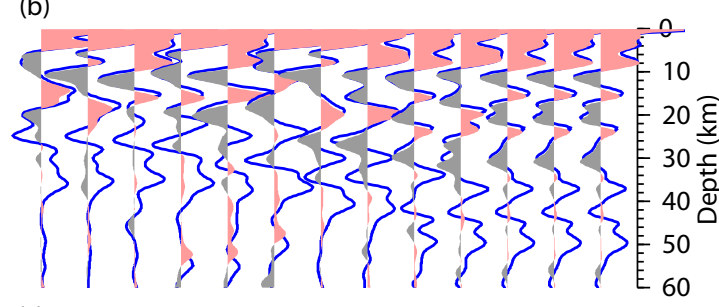

(c)

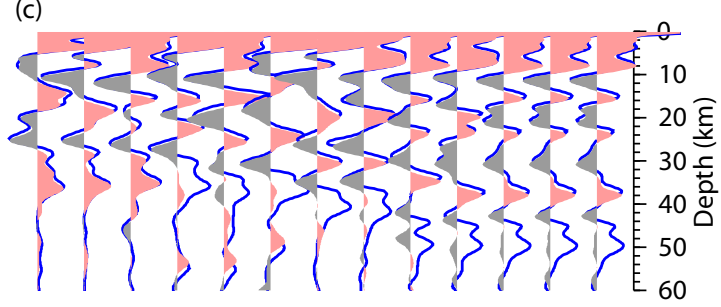

(d)

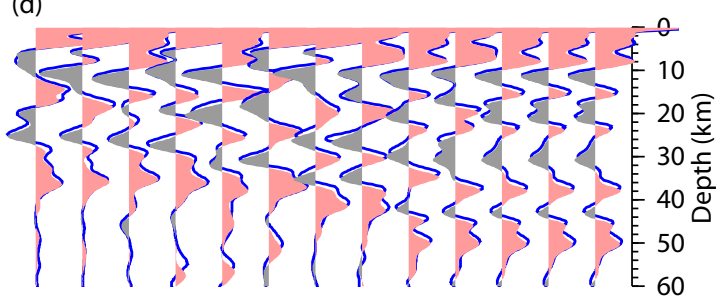

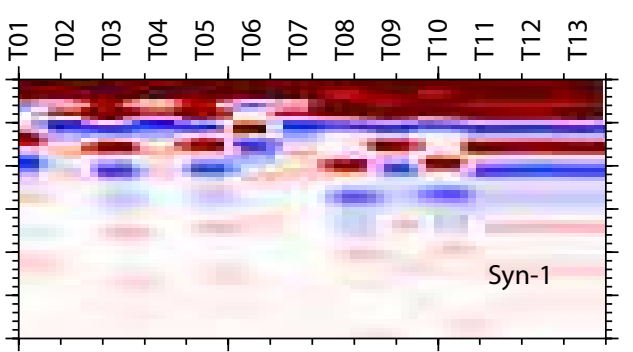
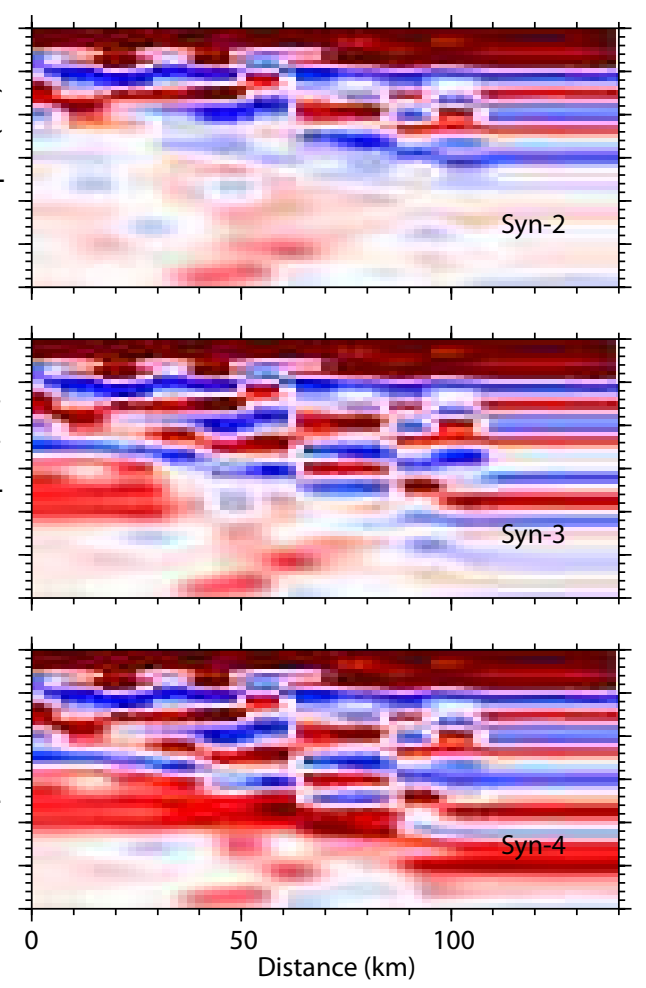

Figure 5. The stepwise inversion results for Model B, including (a) step-one inversion results, (b) step-two inversion results, (c) step-three inversion results, and (d) final inversion results. Left panels show the comparison between $O(z)$ (blue lines) and $Y(z)$ (marked in pink and gray). Right panels show the corresponding synthetic CCP images. Station names are labeled on the top of the plots. Only three inversion steps were performed for stations T01, T02, and T03, and their results in (d) are the same as those in (c). 
tral distances in the range of $39^{\circ}-67^{\circ}$ and back azimuths within the range of $41^{\circ}-70^{\circ}$ (Table S2). The CCP stacking image of the test system for Model $B$ is shown in Figure $4 \mathrm{~b}$. The $O(z)$ obtained from the test system for Model $B$ and the best-fit $Y(z)$ resulting from the CCA inversion are shown in Figure $4 c$, in which the resulting models are used as the migration models. The resulting $V_{\mathrm{s}}$ models are shown in Figure $4 \mathrm{~d}$ and compared with the test models.

Stepwise inversion from the surface down is a key technique to avoid multiple wave interference. We take Model B as an example to describe the stepwise inversion processes in detail. The estimation of reliable structure model frames during inversion is also described accordingly. The first step of inversion (Figure 5a) estimates the velocity structure of the sedimentary cover, including $V_{\mathrm{s}}, V_{\mathrm{p}} / V_{\mathrm{s}}$ and the thickness of each layer. The first positive signals of the CCA image define the depth range of the sedimentary cover (Figure 4c). In this numerical experiment, the minimum thickness of a layer is always set to 0 , while the maximum depth of the basement interface exceeds the depth range of the first positive signals of the CCA. The parameter $V_{\mathrm{s}}$ ranges from $1.7 \mathrm{~km} / \mathrm{s}$ to $3.2 \mathrm{~km} / \mathrm{s}$, and $V_{\mathrm{p}} / V_{\mathrm{s}}$ is set to vary from an upper limit of 2.4 near the surface to a lower limit of 1.75 for the sediments. Sediment related signals in $Y(z)$ can be traced from the surface to a depth of more than $10 \mathrm{~km}$ (Figures 4 and 5a), although the sedimentary cover is less than $5 \mathrm{~km}$ thick (Table 2). The signals below the sedimentary cover, which are generated by multiples, clearly cannot be identified as velocity discontinuities in the subsequent inversion. Synthetic CCP imaging Syn-1 (Figure 5a) is calculated based on the resulting sedimentary cover models estimated from step one inversion overlying a modified IASP91 model (Kennett and Engdahl, 1991).

We estimate the layered crustal medium below the sedimentary cover according to the polarity changes in the signal of the CCA. The parameter $V_{\mathrm{s}}$ ranges from $3.0 \mathrm{~km} / \mathrm{s}$ to $4.2 \mathrm{~km} / \mathrm{s}$. The parameter range of $V_{p} / V_{s}$ is set to vary from 1.72 to 1.78 . The minimum thickness of a layer is set to 0 , while the maximum thickness of a layer is set to $5-15 \mathrm{~km}$ according to the status of the layer in the observational CCA (Figure 4c). To distinguish the multiples from the intracrustal interfaces, we iteratively run the inversion process. Layers L3 and L4, layers L5 and L6, and layers L7-L10 are determined by the second (Figure $5 b$ ), third (Figure $5 c$ ), and fourth step (Figure $5 d$ ) operations of the CCA inversion, respectively. One of the advantages of the CCA inversion method is the self-regulation of the migration model. $O(z)$ is also adjusted at each iteration of the search scheme by using the corresponding accepted model as the migration model. Better fitting between $Y(z)$ and $O(z)$ is gradually obtained in the stepwise inversion.

\section{Discussion on Resolution and Limitations of CCA Inversion}

We compare the resulting model parameters $\left(V_{s}, V_{p} / V_{s}\right.$, and

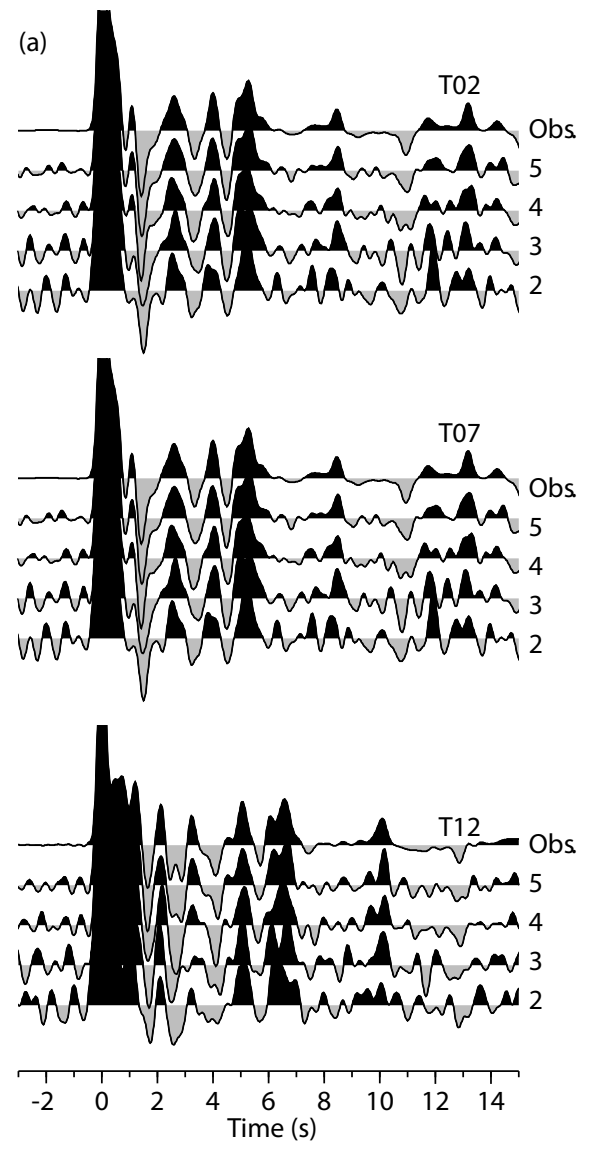

(b)
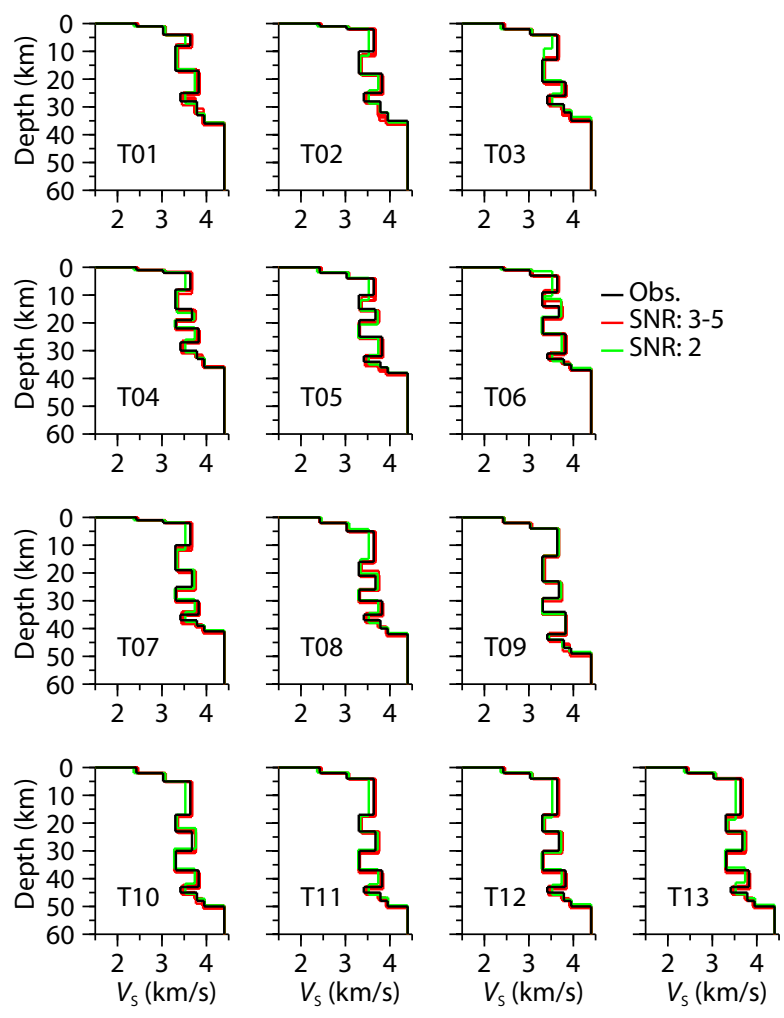

Figure 6. (a) Example of synthetic receiver functions with noise at stations T02, T07, and T12. The value of SNR is shown on the right. (b) Comparison between the $V_{s}$ velocity models obtained from the test system (black lines) and resulting from the CCA inversion with noise interference (red and green lines). The station names are labeled in the graphs. 
depths of interfaces) obtained from the CCA inversion with those from the test models to confirm the reliability of the CCA inversion. The velocity parameters of the test models and the resulting models obtained from the CCA inversion are listed in Tables 1 and 2. The comparison of $V_{\mathrm{s}}$ models between the resulting models and test models is also shown in Figures $3 \mathrm{~d}$ and $4 \mathrm{~d}$. The relative error ranges of $V_{s}$ are $0.0-0.9 \%$ for Model $A$ and $0.3-2.0 \%$ for Model B. The relative error ranges of $V_{\mathrm{p}} / V_{\mathrm{s}}$ are $0.2-1.5 \%$ for Model $\mathrm{A}$ and $0-4.0 \%$ for Model $B$. The maximum relative error range of interface depth is $4.1 \%$ for Model A and $9.2 \%$ for Model B. These small error ranges demonstrate the validity of the CCA inversion methodfor imaging complex crustal structures. Numerical experiments show that the CCA inversion can reconstruct both shear wave velocities and interface depths in complex structures. The performance of CCA is superior to that of conventional receiver function waveform analysis.

Teleseismic recordings usually contain seismic noise due to the conditions of the site and the instrument. We check the stability of the CCA inversion by adding Gaussian white noise into the synthetic vertical and radial components generated by test Model B, and both components have the same signal-to-noise ratio (SNR). The corresponding receiver function is thus described by the same SNR (Figure 6a). As shown in Figure 6b, even if the SNR of the noise model is as low as 2 , reliable results can be obtained from the CCA inversion, and the relative error of $V_{s}$ is generally less than $3.2 \%$.

In the CCA inversion, the model framework and the range of search parameters are the prior control factors. Since the thickness range starts from 0 , it is acceptable to be equal to or greater
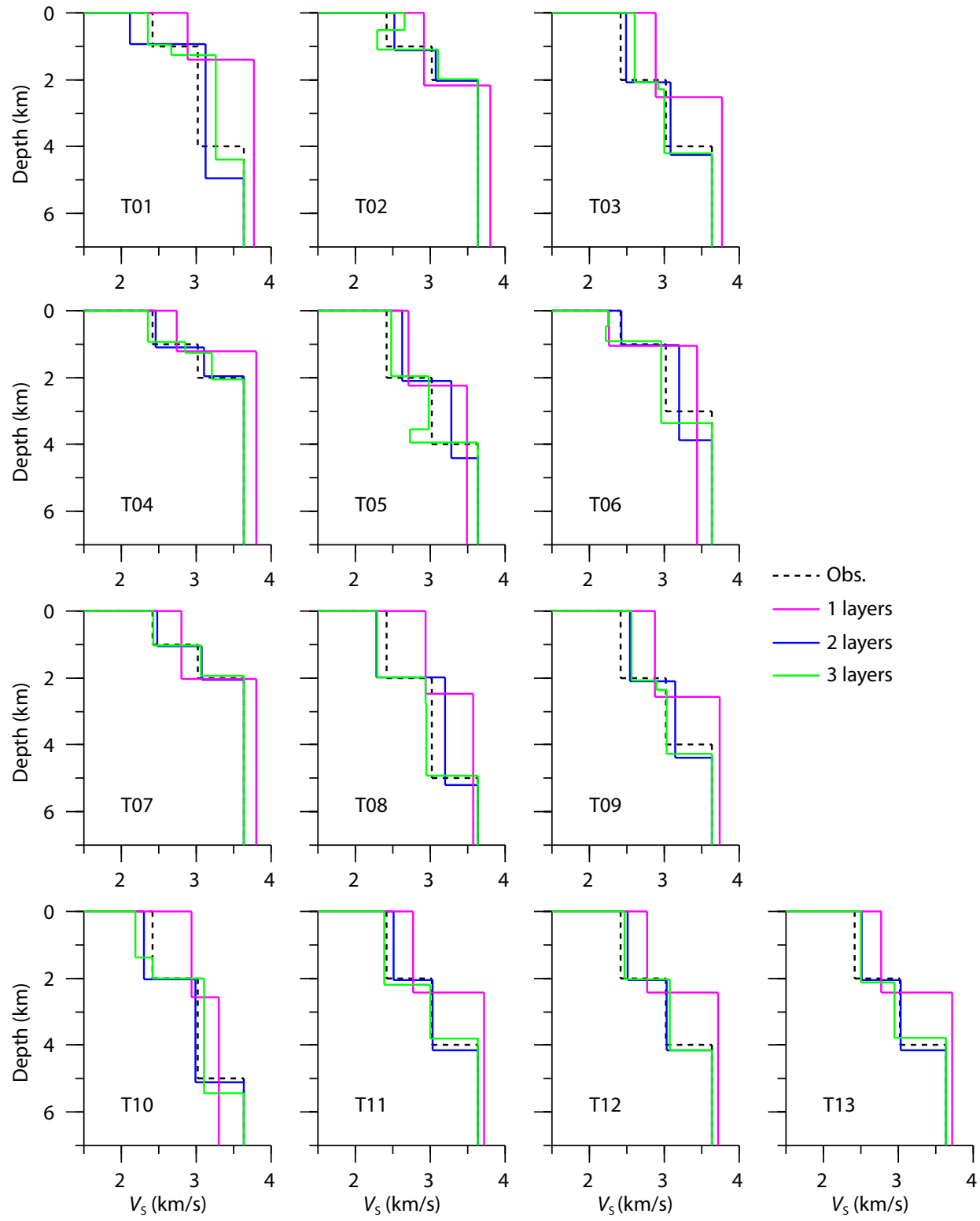

Figure 7. Comparison between the $V_{\mathrm{s}}$ velocity models obtained from the test system (black dashed lines) and resulting from the CCA inversion with 1 layer (purple lines), 2 layers (blue lines) or 3 layers (green lines). 
than the actual number of layers. Figure 7 is an example of using different numbers of layers to invert the sedimentary cover of Model B. An inversion with less than the actual number of layers will inevitably cause distortion.

\section{An Application Example}

As an application example of the CCA inversion method, we measure the $V_{s}$ and $V_{p} / V_{s}$ of crustal layers along a southeastern section of the NCISP6 profile (ES-NCISP6), which crosses the Liaodong Peninsula, Northeast China (Figure 8). The dominant low-velocity structure revealed from receiver function waveform inversion provides information on crustal evolution (Figure 9b, Zheng TY et al., 2015). However, in previous imaging of crustal structure, the velocity values were preset according to the average crustal model of North China, and only the interfaces were obtained by the waveform inversion of the receiver function. In this study, the CCA inversion is used to estimate the properties of low-velocity media by determining the values of $V_{s}$ and $V_{p} / V_{s}$ and therefore to constrain the results of crustal rejuvenation in the study area.

According to the data analysis and CCP imaging of ES-NCISP6 (Zheng TY et al., 2015), we select high-quality receiver functions of 15 events with epicentral distances in the range of $38^{\circ}-83^{\circ}$ observed in the dominant northeast azimuth $\left(40^{\circ}-55^{\circ}\right)$ at 15 stations for the CCA inversion. The station locations are listed in Table S3. The earthquake catalog is listed in Table S4. Similar to the previous inversion of Model B, from sedimentary cover, upper crust, and lower crust to the crust mantle transition zone, the velocity inversion of each station is completed in four steps. The search range of $V_{s}$ is estimated based on the S-wave velocity of northeastern China from joint inversion of the Rayleigh wave phase and group velocities (Li YH et al., 2012). The resulting crustal $V_{\mathrm{s}}$ structure is shown in Figure 9a. The best-fit synthetic CCA agrees well with the observed CCA and is clearly improved compared with those calculated from the previous receiver function waveform inversion (Zheng TY et al., 2015) (Figures 9c and 9d).

Since the velocity of each layer was preset, the previous waveform inversion resulted in a relatively smooth crustal structure. The significant lateral heterogeneity of the $V_{s}$ distribution revealed in this study seems to be more consistent with geological observations. ES-NCISP6 crosses the Paleoproterozoic Liaoji orogenic belt, which experienced intense extensional deformation and magmatism in the Late Jurassic and Early Cretaceous. The Paleoproterozoic Liaohe Group is exposed in the western Liaodong Peninsula (Figure 8), where a lower crustal medium with $V_{\mathrm{s}}$ of 3.64 $\mathrm{km} / \mathrm{s}$ has been uplifted near the surface. Granites are distributed in the middle and east (Figure 8). The lower-velocity medium with $V_{\mathrm{s}}$ of $3.30 \mathrm{~km} / \mathrm{s}$ occupies the lower crust of the two regions, which may imply the deep genesis of granites. However, due to the relatively low velocity, another explanation may also need to be considered, that is, delamination and magmatism caused by the $\mathrm{Pa}$ cific plate subduction since the Cenozoic. The Wulong gold ore concentration district is located in the east (Figure 8). The space occupied by magma in the crust revealed by the new imaging results might provide a deep structural background for gold mineralization on the Liaodong Peninsula.

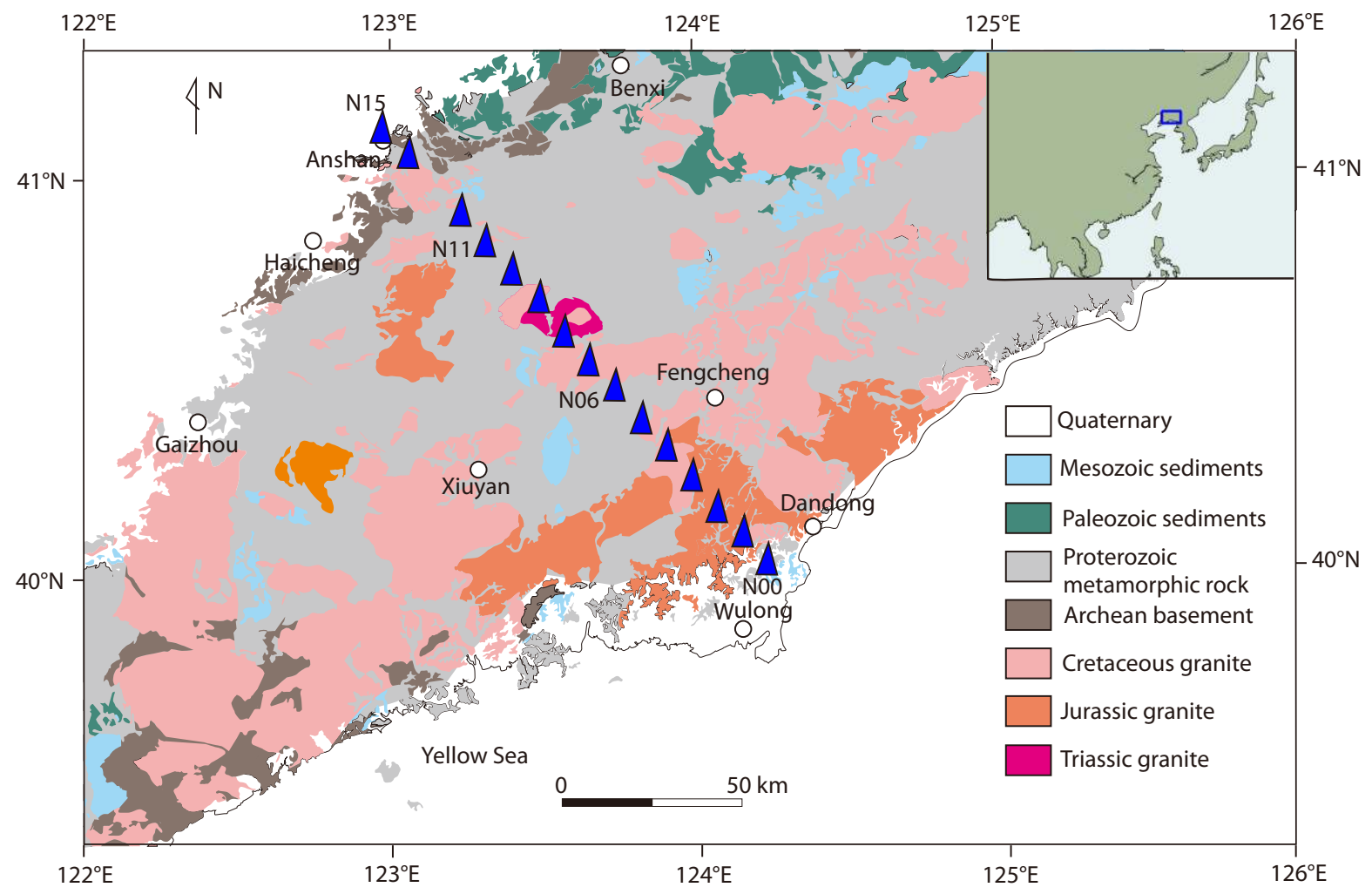

Figure 8. Regional geological map of the Liaodong Peninsula, Northeast China (from Zeng QD et al., 2019) with seismic stations (blue triangles) used in this study. The inset shows the location of the study region in eastern Asia. 


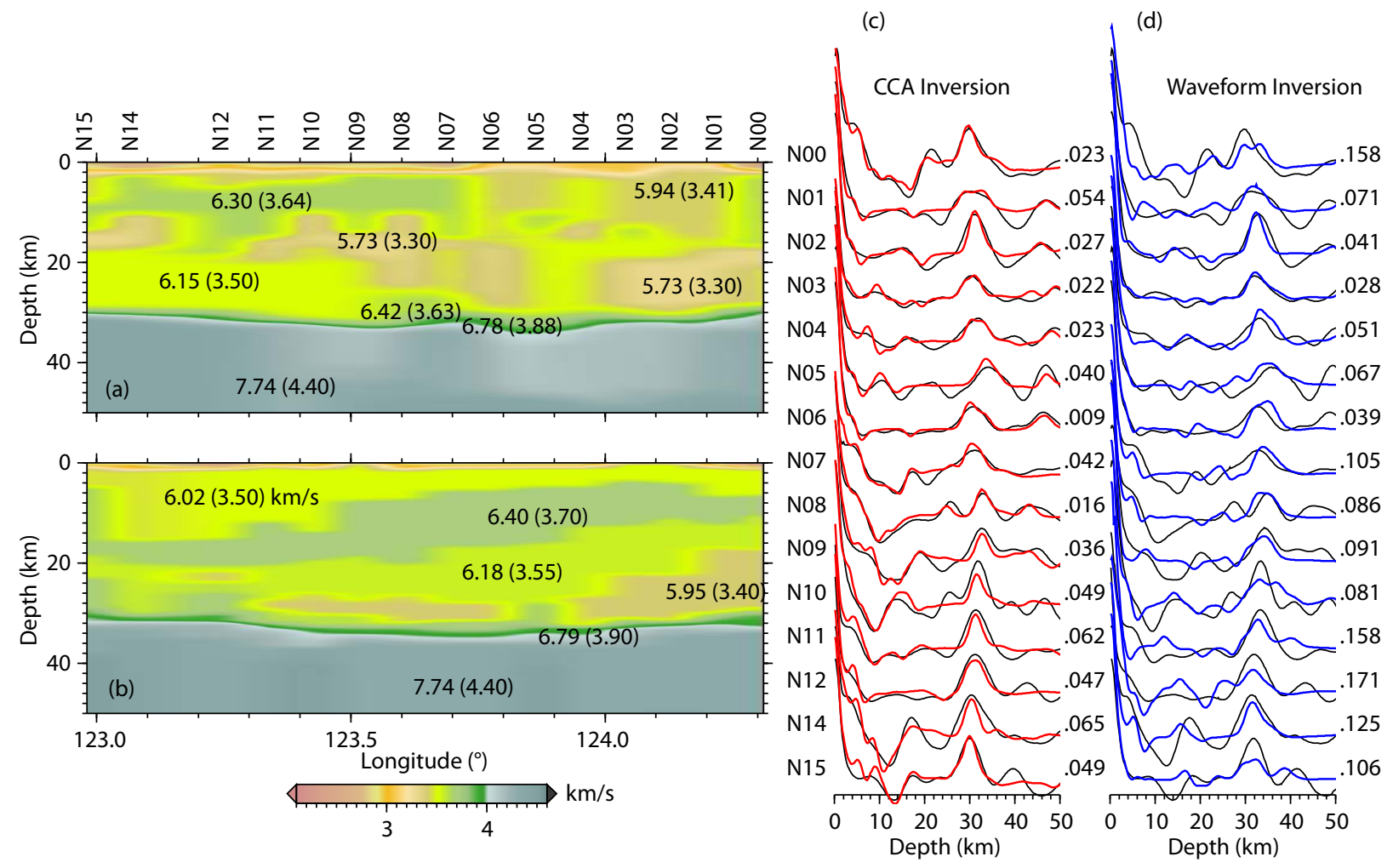

Figure 9. Comparison of crustal $V_{s}$ structures resulting from (a) the CCA inversion and (b) the previous waveform inversion. The values of $V_{s}$ are indicated by the color bar at the bottom of the plots. Station names are labeled at the top of the plots. (c) Comparison between $O(z)$ (black lines) and $Y(z)$ (red lines) resulting from the CCA inversion. (d) Comparison between $O(z)$ (black lines) and $Y(z)$ (blue lines) resulting from the crustal $V_{s}$ structures obtained by previous receiver function waveform inversion. Numbers on the right indicate the value of $O B J$ calculated using $O(z)$ and $Y(z)$ in the depth range of $0-40 \mathrm{~km}$. Station names are labeled on the left side of the plots.

\section{Conclusions}

To obtain the detailed crustal structure from the receiver function, we develop the CCA inversion method in the depth domain. This approach could efficiently suppress the shortcoming of easily falling into the local extremum in conventional waveform inversion. Numerical tests document the ability and reliability of the method in estimating the absolute $V_{\mathrm{s}}, V_{\mathrm{p}} / V_{\mathrm{s}}$, and layer thickness in a region with a complex crustal structure. The application example shows that the crustal structure can be much better reconstructed than by receiver function waveform inversion, and fine crustal velocity structures would provide significant information on subsequent tectonic studies. The technical principle of the CCA inversion method can be extended to the structural imaging of a threedimensional observation system. Meanwhile, the development of nonlinear inversion techniques and synthetic imaging methods would contribute to the improvement of the CCA method in the future.

\section{Acknowledgments}

This study was financially supported by the National Natural Science Foundation of China (Grant 91755214).

\section{References}

Ai, Y. S., Liu, P. C., and Zheng, T. Y. (1998). Adaptive hybrid global inversion algorithm. Sci. China Ser. D-Earth Sci., 41(2), 137-143.
https://doi.org/10.1007/BF02932432

Ammon, C. J., Randall, G. E., and Zandt, G. (1990). On the nonuniqueness of receiver function inversions. J. Geophys. Res. :Solid Earth, 95(B10), 15303-15318. https://doi.org/10.1029/JB095iB10p15303

Chang, S. J., Baag, C. E., and Langston, C. A. (2004). Joint analysis of teleseismic receiver functions and surface wave dispersion using the Genetic algorithm. Bull. Seismol. Soc. Am., 94(2), 977-987. https://doi.org/10.1785/0120030110

Chen, L., Wen, L. X., and Zheng, T. Y. (2005). A wave equation migration method for receiver function imaging: 1. Theory. J. Geophys. Res. :Solid Earth, 110(B11), B11309. https://doi.org/10.1029/2005JB003665

Chen, L., Zheng, T. Y., and Xu, W. W. (2006). A thinned lithospheric image of the Tanlu Fault Zone, eastern China: Constructed from wave equation based receiver function migration. J. Geophys. Res. :Solid Earth, 111 (B9), B09312. https://doi.org/10.1029/2005JB003974

Dueker, K. G., and Sheehan, A. F. (1997). Mantle discontinuity structure from midpoint stacks of converted $P$ to $S$ waves across the Yellowstone hotspot track. J. Geophys. Res. :Solid Earth, 102(B4), 8313-8327. https://doi.org/10.1029/96JB03857

Ford, H. A., Fischer, K. M., Abt, D. L., Rychert, C. A., and Elkins-Tanton, L. T. (2010). The lithosphere-asthenosphere boundary and cratonic lithospheric layering beneath Australia from Sp wave imaging. Earth Planet. Sci. Lett., 300(3-4), 299-310. https://doi.org/10.1016/j.epsl.2010.10.007

Gilbert, H. J., Sheehan, A. F., Dueker, K. G., and Molnar, P. (2003). Receiver functions in the western United States, with implications for upper mantle structure and dynamics. J. Geophys. Res. :Solid Earth, 108(B5), 2229. https://doi.org/10.1029/2001JB001194

He, Y. M. , Zheng, T. Y. , Ai, Y. S. , Hou, G. B. , and Chen, Q. F. (2018). Growth of the lower continental crust via the relamination of arc magma. Tectonophysics, 724-725, 42-50.https://doi.org/10.1016/j.tecto.2018.01.006 
https://doi.org/10.1016/j.tecto.2018.01.006

Julià, J., Ammon, C. J., Herrmann, R. B., and Correig, A. M. (2000). Joint inversion of receiver function and surface wave dispersion observations. Geophys. J. Int., 143(1), 99-112. https://doi.org/10.1046/j.1365-246x.2000.00217.x

Kennett, B. L. N. (1983). Seismic Wave Propagation in Stratified Media. Cambridge: Cambridge University Press.

Kennett, B. L. N. , and Engdahl, E. R. 1991. Traveltimes for global earthquake location and phase identification. Geophys. J. Int. , 105(2), 429465.https://doi.org/10.1111/j.1365-246X.1991.tb06724.x https://doi.org/10.1111/j.1365-246X.1991.tb06724.x

Kind, R., Yuan, X., Saul, J., Nelson, D., Sobolev, S. V., Mechie, J., Zhao, W., Kosarev, G., Ni, J., ... Jiang, M. (2002). Seismic images of crust and upper mantle beneath Tibet: Evidence for Eurasian plate subduction. Science, 298(5596), 1219-1221. https://doi.org/10.1126/science.1078115

Langston, C. A. (1979). Structure under Mount Rainier, Washington, inferred from teleseismic body waves. J. Geophys. Res. :Solid Earth, 84(B9), 4749-4762. https://doi.org/10.1029/JB084iB09p04749

Lawrence, J. F., and Shearer, P. M. (2006). A global study of transition zone thickness using receiver functions. J. Geophys. Res. :Solid Earth, 111, B06307. https://doi.org/10.1029/2005JB003973

Li, Y. H., Wu, Q. J., Pan, J. T., and Sun, L. (2012). S-wave velocity structure of northeastern China from joint inversion of Rayleigh wave phase and group velocities. Geophys. J. Int., 190(1), 105-115. https://doi.org/10.1111/j.1365246X.2012.05503.x

Ling, Y., Zheng, T. Y., He, Y. M., and Hou, G. B. (2020). Response of Yunnan crustal structure to eastward growth of the Tibet Plateau and subduction of the India plate in Cenozoic. Tectonophysics, 797, 228661. https://doi.org/10.1016/j.tecto.2020.228661

Liu, P. C., Hartzell, S., and Stephenson, W. (1995). Non-linear multiparameter inversion using a hybrid global search algorithm: applications in reflection seismology. Geophys. J. Int., 122(3), 991-1000. https://doi.org/10.1111/j.1365-246X.1995.tb06851.x
Poppeliers, C., and Pavlis, G. L. (2003). Three-dimensional, prestack, plane wave migration of teleseismic P-to-S converted phases: 1. Theory. J. Geophys. Res. :Solid Earth, 108(B2), 2112. https://doi.org/10.1029/2001JB000216

Sheehan, A. F., Shearer, P. M., Gilbert, H. J., and Dueker, K. G. (2000). Seismic migration processing of $P$-SV converted phases for mantle discontinuity structure beneath the Snake River Plain, western United States. J. Geophys. Res. :Solid Earth, 105(B8), 19055-19065. https://doi.org/10.1029/2000JB900112

Tao, K., Niu, F. L., Ning, J. Y., Chen, Y. S., Grand, S., Kawakatsu, H., Tanaka, S., Obayashi, M., and Ni, J. (2014). Crustal structure beneath NE China imaged by NECESSArray receiver function data. Earth Planet. Sci. Lett., 398, 48-57. https://doi.org/10.1016/j.epsl.2014.04.043

Wu, Q. J., and Zeng, R. S. (1998). The crustal structure of Qinghai-Xizang plateau inferred from broadband teleseismic waveform. Acta Geophys. Sin., 41(5), 669-679.

Zeng, Q. D., Chen, R. Y., Yang, J. H., Sun, G. T., Yu, B., Wang, Y. B., and Chen, P. W. (2019). The metallogenic characteristics and exploring ore potential of the gold deposits in eastern Liaoning Province. Acta Petrol. Sin., 35(7), 1939-1963. https://doi.org/10.18654/1000-0569/2019.07.01

Zheng, T. Y., Zhao, L., and Zhu, R. X. (2009). New evidence from seismic imaging for subduction during assembly of the North China craton. Geology, 37(5), 395-398. https://doi.org/10.1130/G25600A.1

Zheng, T. Y., He, Y. M., Yang, J. H., and Zhao, L. (2015). Seismological constraints on the crustal structures generated by continental rejuvenation in northeastern China. Sci. Rep., 5, 14995. https://doi.org/10.1038/srep14995

Zheng, T. Y., He, Y. M., Ding, L., Jiang, M. M., Ai, Y. S., Mon, C. T., Hou, G. B., Sein, K., and Thant, M. (2020). Direct structural evidence of Indian continental subduction beneath Myanmar. Nat. Commun., 11, 1944. https://doi.org/10.1038/s41467-020-15746-3

Zhu, L. P. (2000). Crustal structure across the San Andreas Fault, Southern California from teleseismic converted waves. Earth Planet. Sci. Lett., 179(1), 183-190. https://doi.org/10.1016/S0012-821X(00)00101-1 


\section{Supplementary Materials for "A new approach for inversion of receiver function for crustal atructure in the depth domain"}

This dataset contains earthquake catalogs and basic station information used in this study. The earthquake catalogs used in Model A and Model B of the test system are listed in Tables S1 and S2, respectively. The station information and the earthquake catalog applied in the ES-NCISP6 imaging are shown in Tables S3 and S4.

Table S1. Earthquake catalog used in Model A of the test system.

\begin{tabular}{|c|c|c|c|c|}
\hline \multirow{2}{*}{ Data } & \multicolumn{2}{|c|}{ Location } & \multirow{2}{*}{$\begin{array}{c}\text { Epicenter } \\
\text { distance }\left({ }^{\circ}\right)\end{array}$} & \multirow{2}{*}{$\begin{array}{l}\text { Azimuth } \\
\text { range }\left({ }^{\circ}\right)\end{array}$} \\
\hline & Lat. $\left({ }^{\circ}\right)$ & Long. $\left({ }^{\circ}\right)$ & & \\
\hline 20130223 & $10.66 \mathrm{~S}$ & $165.30 \mathrm{E}$ & $73.3-73.7$ & $109.9-110.2$ \\
\hline 20130201 & $10.63 \mathrm{~S}$ & 166.37E & $74.2-74.6$ & $109.3-109.7$ \\
\hline 20130212 & $10.90 \mathrm{~S}$ & $164.26 \mathrm{E}$ & $72.5-73.0$ & $110.6-111.0$ \\
\hline 20121215 & $4.63 \mathrm{~S}$ & $153.02 E$ & $59.8-60.2$ & $110.9-111.6$ \\
\hline 20130704 & $7.03 \mathrm{~S}$ & $155.73 \mathrm{E}$ & $63.3-63.8$ & $111.7-112.2$ \\
\hline 20121115 & $3.11 \mathrm{~S}$ & 148.27E & $55.0-55.4$ & $112.4-113.2$ \\
\hline 20121202 & $16.98 \mathrm{~S}$ & 167.45E & $78.4-78.9$ & $114.4-114.6$ \\
\hline 20111105 & $18.24 \mathrm{~S}$ & $168.30 \mathrm{E}$ & $79.5-80.1$ & $115.2-115.4$ \\
\hline 20121012 & $4.89 \mathrm{~S}$ & 134.03E & $44.6-45.3$ & $125.4-126.4$ \\
\hline 20121210 & $6.53 \mathrm{~S}$ & 129.83E & $42.7-43.5$ & $131.3-132.3$ \\
\hline 20130223 & $8.47 \mathrm{~S}$ & $127.46 \mathrm{E}$ & $42.6-43.5$ & $135.8-136.7$ \\
\hline 20130708 & $8.80 \mathrm{~S}$ & $127.00 \mathrm{E}$ & $42.5-43.4$ & $136.6-137.5$ \\
\hline
\end{tabular}

Table S2. Earthquake catalog used in Model B of the test system.

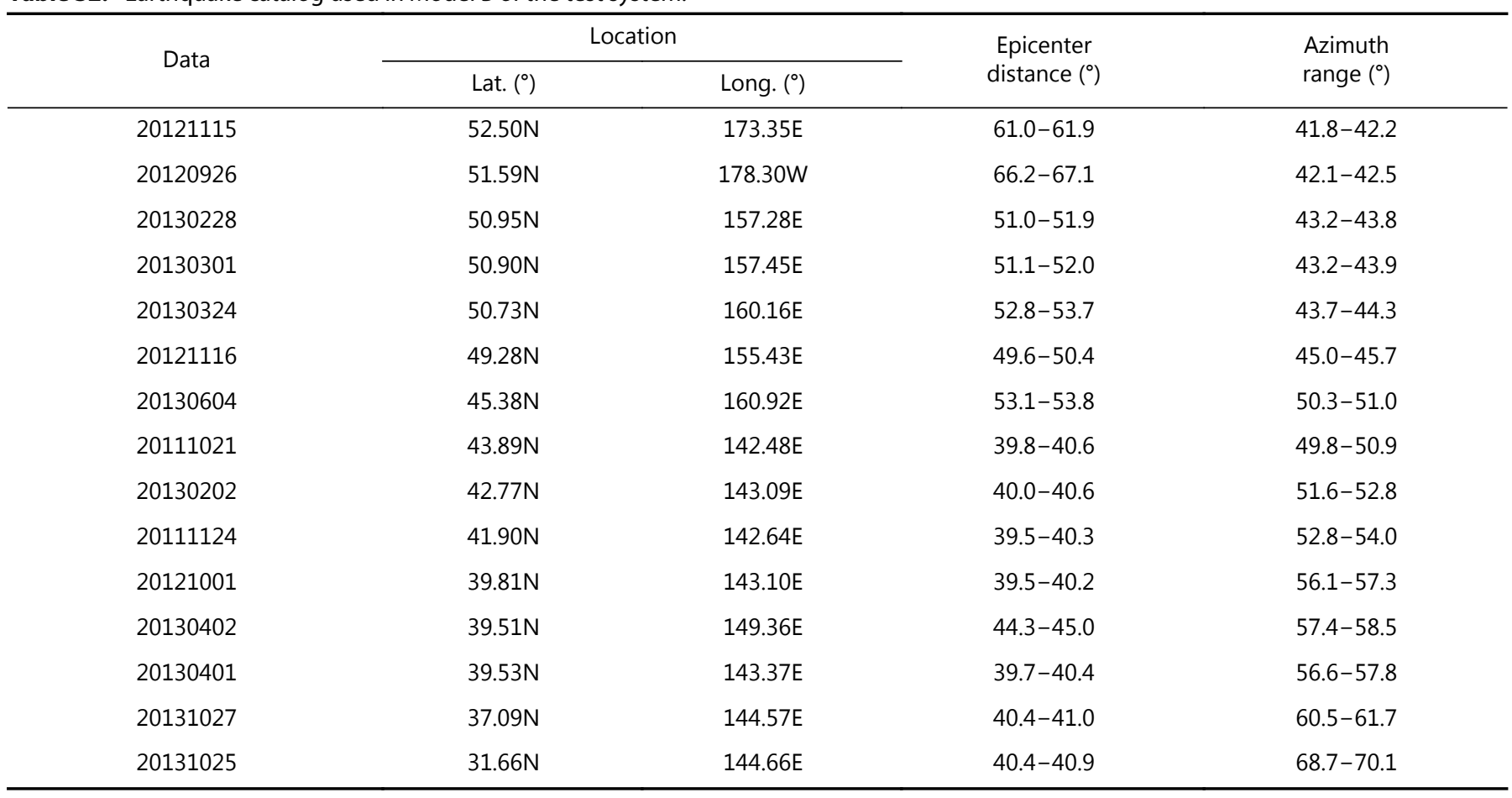


Table S3. Station site details of the ES-NCISP6.

\begin{tabular}{cccc}
\hline Station & Lat. $\left(^{\circ}\right)$ & Long. $\left({ }^{\circ}\right)$ & Number of receiver functions \\
\hline 00 & $40.00 \mathrm{~N}$ & $124.32 \mathrm{E}$ & 16 \\
01 & $40.08 \mathrm{~N}$ & $124.22 \mathrm{E}$ & 8 \\
02 & $40.15 \mathrm{~N}$ & $124.13 \mathrm{E}$ & 24 \\
03 & $40.22 \mathrm{~N}$ & $124.04 \mathrm{E}$ & 15 \\
04 & $40.29 \mathrm{~N}$ & $123.96 \mathrm{E}$ & 26 \\
05 & $40.37 \mathrm{~N}$ & $123.86 \mathrm{E}$ & 18 \\
06 & $40.44 \mathrm{~N}$ & $123.80 \mathrm{E}$ & 20 \\
07 & $40.52 \mathrm{~N}$ & $123.70 \mathrm{E}$ & 15 \\
08 & $40.58 \mathrm{~N}$ & $123.60 \mathrm{E}$ & 10 \\
09 & $40.67 \mathrm{~N}$ & $123.52 \mathrm{E}$ & 28 \\
10 & $40.74 \mathrm{~N}$ & $123.43 \mathrm{E}$ & 23 \\
11 & $40.82 \mathrm{~N}$ & $123.34 \mathrm{E}$ & 16 \\
14 & $40.89 \mathrm{~N}$ & $123.26 \mathrm{E}$ & 22 \\
15 & $41.00 \mathrm{~N}$ & $123.10 \mathrm{E}$ & 16 \\
& $41.05 \mathrm{~N}$ & $123.03 \mathrm{E}$ & 22 \\
\end{tabular}

Table S4. Earthquake catalog used in imaging of ES-NCISP6.

\begin{tabular}{|c|c|c|c|c|c|c|}
\hline \multirow{2}{*}{ Data } & \multicolumn{2}{|c|}{ Location } & \multirow{2}{*}{ Depth (km) } & \multirow{2}{*}{ Magnitude } & \multirow{2}{*}{ Epicenter distance $\left({ }^{\circ}\right)$} & \multirow{2}{*}{ Azimuth range $\left({ }^{\circ}\right)$} \\
\hline & Lat. $\left({ }^{\circ}\right)$ & Long. $\left({ }^{\circ}\right)$ & & & & \\
\hline 08052013 & $51.43 \mathrm{~N}$ & 178.35E & 40 & 6.1 & $38.6-38.8$ & $54.4-55.3$ \\
\hline 08041605 & $52.16 \mathrm{~N}$ & $179.12 W$ & 13 & 6.7 & $38.8-39.9$ & $53.0-53.9$ \\
\hline 08072500 & $51.25 \mathrm{~N}$ & 179.03E & 23 & 6.0 & $39.0-39.2$ & $54.6-55.4$ \\
\hline 07121909 & $51.34 \mathrm{~N}$ & $179.52 W$ & 29 & 6.9 & $39.9-40.1$ & $54.3-55.1$ \\
\hline 07122107 & $51.53 \mathrm{~N}$ & 179.11W & 25 & 6.0 & $40.2-40.2$ & $54.0-54.9$ \\
\hline 08032221 & $52.63 \mathrm{~N}$ & $178.49 \mathrm{~W}$ & 132 & 6.4 & $40.4-40.6$ & $52.2-53.0$ \\
\hline 08050201 & $52.14 \mathrm{~N}$ & $178.11 \mathrm{~W}$ & 16 & 7.0 & $40.7-40.9$ & $52.9-53.8$ \\
\hline 07103113 & $51.53 \mathrm{~N}$ & $177.97 \mathrm{~W}$ & 30 & 6.3 & $40.9-41.0$ & $53.8-54.6$ \\
\hline 08052519 & $55.90 \mathrm{~N}$ & $153.50 \mathrm{~W}$ & 10 & 6.4 & $53.9-54.0$ & $43.6-43.8$ \\
\hline 08010511 & $51.30 \mathrm{~N}$ & $130.80 \mathrm{~W}$ & 10 & 7.0 & $67.9-68.0$ & $40.7-40.9$ \\
\hline 08011001 & $43.80 \mathrm{~N}$ & $127.30 \mathrm{~W}$ & 10 & 6.4 & 74.5 & 45.2 \\
\hline 08022114 & $41.20 \mathrm{~N}$ & $114.90 \mathrm{~W}$ & 6 & 6.4 & $82.8-82.9$ & $40.3-40.7$ \\
\hline
\end{tabular}

